\title{
Edge Coloring and Decompositions of Weighted Graphs
}

\author{
Uriel Feige*and Mohit Singh ${ }^{\dagger}$
}

June 25, 2008

\begin{abstract}
We consider two generalizations of the edge coloring problem in bipartite graphs. The first problem we consider is the WEIGHTED BIPARTITE EDGE COLORING problem where we are given an edge-weighted bipartite graph $G=(V, E)$ with weights $w: E \rightarrow[0,1]$. The task is to find a proper weighted coloring of the edges with as few colors as possible. An edge coloring of the weighted graph is called a proper weighted coloring if the sum of the weights of the edges incident to a vertex of any color is at most one. We give a polynomial time algorithm for the WEIGHTED BIPARTITE EDGE COLORING problem which returns a proper weighted coloring using at most $\lceil 2.25 n\rceil$ colors where $n$ is the maximum total weight incident at any vertex. This improves on the previous best bound of Correa and Goemans [5] which returned a coloring using $2.557 n+o(n)$ colors. The second problem we consider is the BALANCED DECOMPOSITION OF BIPARTITE GRAPHS problem where we are given a bipartite graph $G=(V, E)$ and $\alpha_{1}, \ldots, \alpha_{k} \in(0,1)$ summing to one. The task is to find a partition $E_{1}, \ldots, E_{k}$ of $E$ such that $\operatorname{deg}_{E_{i}}(v)$ is close to $\alpha_{i} \operatorname{deg}_{E}(v)$ for each $1 \leq i \leq k$ and $v \in V$. We give an alternate proof of the result of Correa and Goemans [5] that there is a decomposition such that $\left\lfloor\alpha_{i} \operatorname{deg}_{E}(v)\right\rfloor-2 \leq \operatorname{deg}_{E_{i}}(v) \leq\left\lceil\alpha_{i} \operatorname{deg}_{E}(v)\right\rceil+2$ for each $v \in V$ and $1 \leq i \leq k$. Moreover, we show that the additive error can be improved from two to one if only upper bounds or only lower bounds on the degree are present. All our results hold also for bipartite multigraphs, and the decomposition results hold also for general graphs.
\end{abstract}

\footnotetext{
*Weizmann Institute, Rehovot, Israel. uriel.feige@weizmann.ac.il

†Tepper School of Business, Carnegie Mellon University, Pittsburgh, USA. mohits@andrew.cmu.edu.
} 


\section{Introduction}

Edge coloring problems have been crucial in the development of different algorithmic techniques and have also been used to model various scheduling problems. In this paper, we consider two edge coloring problems which have been inspired from study of Clos networks [4] and also generalize classical coloring problems. Clos network were introduced by Clos [4] in the context of designing interconnection networks used to route telephone calls and have found various applications $[2,10]$. We refer the reader to Correa and Goemans [5] for the relationship between the problems considered here and Clos networks.

The first problem we consider is the WEIGHTED BIPARTITE EDGE COLORING problem where we are given an edge-weighted bipartite graph $G=(V, E)$ with weights $w: E \rightarrow[0,1]$. The task is to find a proper weighted coloring of the edges with as few colors as possible. An edge coloring of the weighted graph is called a proper weighted coloring if the sum of the weights of the edges incident to a vertex of any color is at most one. If all the edges have weight one then the problem reduces to the classical bipartite edge coloring problem. König's Theorem [12] gives an optimal coloring in this case where the number of colors used is exactly the maximum degree of the graph. For the weighted bipartite edge coloring problem, Chung and Ross [3] gave the following conjecture.

Conjecture 1.1 Given an instance of the WEIGHTED BIPARTITE EDGE COLORING problem, there is a proper weighted coloring using at most $2 n-1$ colors where $n$ denotes the maximum over all the vertices of the number of unit-sized bins needed to pack the weights of edges incident at the vertex.

The following is a stronger version of the Conjecture 1.1.

Conjecture 1.2 Given an instance of the WEIGHTED BIPARTITE EDGE COLORING problem, there is a proper weighted coloring using at most $2 n-1$ colors where $n$ is the smallest integer greater than the maximum over all the vertices of the total weight of edges incident at the vertex.

Conjecture 1.2 is the best possible since there are instances where any proper weighted coloring takes $2 n-1$ colors. Melen and Turner [15] showed that the Conjecture 1.2 is true when all edge-weights are at most $\frac{1}{2}$. Moreover when all weights are strictly more than $\frac{1}{2}$, Conjecture 1.2 is also true and follows simply from König's Theorem.

One of our main results in the paper makes progress towards the resolution of Conjecture 1.2 and therefore Conjecture 1.1.

Theorem 1.3 There is a polynomial time algorithm for the WEIGHTED BIPARTITE EDGE COLORING problem which returns a proper weighted coloring using at most $\lceil 2.25 n\rceil$ colors where $n$ is the maximum total weight incident at any vertex.

Theorem 1.3 improves on the previous best result given by Correa and Goemans [5] who give a coloring using at most $2.557 n+o(n)$ colors. Correa and Goemans [5] also give an algorithm which returns a proper weighted coloring with $2.5480 n+o(n)$ colors where $n$ denotes the maximum over all the vertices of the number of unit-sized bins needed to pack the weights of incident edges. Theorem 1.3 implies the improved bound of $\lceil 2.25 n\rceil$ for this variant as well.

The second problem we consider is the BALANCED DECOMPOSITION OF BIPARTITE GRAPHS problem where we are given a bipartite graph $G=(V, E)$ and $\alpha_{1}, \ldots, \alpha_{k} \in(0,1)$ summing to one. The task is to find a partition $E_{1}, \ldots, E_{k}$ of $E$ such that $\operatorname{deg}_{E_{i}}(v)$ is close to $\alpha_{i} \operatorname{deg}_{E}(v)$ for each $1 \leq i \leq k$ and $v \in V$. Correa and Goemans [5] gave the following conjecture. 
Conjecture 1.4 Given an instance of Balanced Decomposition of Bipartite Graph problem there exists a decomposition such that

$$
\left\lfloor\alpha_{i} \operatorname{deg}_{E}(v)\right\rfloor \leq \operatorname{deg}_{E_{i}}(v) \leq\left\lceil\alpha_{i} \operatorname{deg}_{E}(v)\right\rceil
$$

for each $1 \leq i \leq k$ and each $v \in v$.

Correa and Goemans [5] proved a relaxed version of the conjecture in which both the upper and lower bounds are relaxed by two, i.e, the decomposition guarantees that $\left\lfloor\alpha_{i} \operatorname{deg}_{E}(v)\right\rfloor-2 \leq$ $\operatorname{deg}_{E_{i}}(v) \leq\left\lceil\alpha_{i} \operatorname{deg}_{E}(v)\right\rceil+2$.

We give an alternate proof of the result of Correa and Goemans [5] using linear programming methods and then show that the violation can be bounded by an additive one if only upperbound (or lowerbound) are present.

Theorem 1.5 Given an instance of BALANCED Decomposition of Bipartite GRAPH problem there exists a decomposition $E_{1}, \ldots, E_{k}$ of $E$ such that

$$
\left\lfloor\alpha_{i} \operatorname{deg}_{E}(v)\right\rfloor-2 \leq \operatorname{deg}_{E_{i}}(v) \leq\left\lceil\alpha_{i} \operatorname{deg}_{E}(v)\right\rceil+2
$$

for each $1 \leq i \leq k$ and each $v \in V$. Moreover, there are decompositions $F_{1}, \ldots, F_{k}$ and $G_{1}, \ldots G_{k}$ such that

$$
\begin{aligned}
& \operatorname{deg}_{F_{i}}(v) \leq\left\lceil\alpha_{i} \operatorname{deg}_{E}(v)\right\rceil+1 \\
& \operatorname{deg}_{G_{i}}(v) \geq\left\lfloor\alpha_{i} \operatorname{deg}_{E}(v)\right\rfloor-1
\end{aligned}
$$

for each $1 \leq i \leq k$ and $v \in V$.

\subsection{Previous Work}

Two classical results on edge coloring are König's theorem [12] for coloring a bipartite graph with $\Delta$ colors and the Vizing's theorem [18] for coloring any simple graph with $\Delta+1$ colors where $\Delta$ is the maximum degree of the graph.

Before we review some of the existing literature on the problems discussed in this paper, we introduce some notation. Given a weighted bipartite graph $G=(A \cup B, E)$ with weights $w: E \rightarrow[0,1]$, let $\chi_{w}^{\prime}(G)$ denote the minimum number of colors needed to obtain a proper weighted coloring of $G$. Given positive integers $n$ and $r$, let $M(n, r)=\max _{G} \chi_{w}^{\prime}(G)$ where the maximum is taken over all bipartite graphs $G=(A \cup B, E)$ with $|A|=|B|=r$ and $\max _{v \in V} \sum_{e \in \delta(v)} w_{e} \leq n$. In this notation, Conjecture 1.2 can be reformulated to claim that $M(n, r) \leq 2 n-1$.

Given positive integers $n$ and $r$, let $m(n, r)=\max _{G} \chi_{w}^{\prime}(G)$ where the maximum is taken over all bipartite graphs $G=(A \cup B, E)$ with $|A|=|B|=r$ and where $n$ is the maximum over all the vertices of the number of unit-sized bins needed to pack the weights of incident edges. Conjecture 1.1 can be reformulated to claim that $m(n, r) \leq 2 n-1$. It is easy to see that Conjecture 1.2 implies Conjecture 1.1 since $m(n, r) \leq M(n, r)$ for each $n$ and $r$.

If the weight function is restricted to $w: E \rightarrow I$ for some interval $I \subseteq[0,1]$, then we let the minimum number of colors be denoted by $M_{I}(n, r)$ an $m_{I}(n, r)$ respectively. Melen and Turner [15] proved that $m_{[0,1 / 2]}(n, r) \leq M_{[0,1 / 2]}(n, r) \leq 2 n-1$ and in general showed that

$$
m_{[0, B]}(n, r) \leq M_{[0, B]}(n, r) \leq \frac{n}{1-B}
$$

Improving the bounds for $m(n, r)$ and $M(n, r)$ has received considerable attention $[3,5,8$, $15,16,17]$ and the previous best bounds known were 


$$
\begin{gathered}
\frac{5 n}{4} \leq m(n, r) \leq 2.548 n+o(n) \\
2 n-1 \leq M(n, r) \leq 2.557 n+o(n)
\end{gathered}
$$

where both the upper bounds are by Correa and Goemans [5]. The lower bound on $m(n, r)$ is due to $\mathrm{Ngo}$ and $\mathrm{Vu}[16]$ and lower bound on $M(n, r)$ is due to Du et al [8]. Our results improve the upper bounds for both $m(n, r)$ and $M(n, r)$ to $\lceil 2.25 n\rceil$ making progress towards resolution of Conjecture 1.1 and Conjecture 1.2.

The Balanced Decomposition of Bipartite Graphs problem was introduced by Correa and Goemans [5] who proved a relaxed version of the Conjecture 1.4 as mentioned above. Some special cases of Conjecture 1.4 are known to be true. When $k=2$, Conjecture 1.4 is true and the decomposition was given by Hoffman [9]. When each $\alpha_{i}$ is equal to $\frac{1}{k}$ then de Werra [7] showed that the conjecture is true. The conjecture is also true when $G$ is regular or when $\alpha_{i} \operatorname{deg}_{E}(v)$ is an integer for each $i$ and $v$ and follows from König's edge coloring theorem [12].

\subsection{Bipartite Versus General Graphs}

In all results stated in the paper, there is no distinction between bipartite graphs and bipartite multigraphs (allowing parallel edges): the proofs apply without change also to bipartite multigraphs. Conjectures 1.2 and 1.4 do not hold as stated for arbitrary (non-bipartite) graphs. We elaborate on this here, and explain which of the results in this paper extend to general graphs.

Consider the Petersen graph which is a regular graph of degree 3 whose edges cannot be properly colored by 3 colors. Give every edge a weight $2 / 3$. In the setting of Conjecture 1.2 this corresponds to a value of $n=2$ and a requirement for a proper weighted coloring with 3 colors, which is impossible. This shows that when general graphs are concerned, the term $2 n-1$ in the conjecture needs to be raised to at least $2 n$.

If one allows parallel edges, then having odd cycles has a more dramatic effect. Consider for example a triangle with $k$ parallel edges between any two vertices. Give each edge a weight of $(k+1) / 2 k$. Now $n$ corresponds to $k+1$, whereas any proper weighted coloring requires $3 k$ colors. Hence as $k$ grows, the bound in Conjecture 1.1 approaches $3 n$ (if the graph is non-bipartite and has parallel edges).

Applications of Conjecture 1.2 often involve bipartite graphs with parallel edges. And indeed, our proof of Theorem 1.3 works without change even if the bipartite graph has parallel edges. For general (non-bipartite) graphs, our proof of Theorem 1.3 easily extends to give a bound of $\lceil 2.25 n\rceil+r$, where $r$ is the maximum multiplicity of any edge (and in particular, $r=1$ in simple graphs). In counting multiplicity of an edge, one may first merge parallel copies of an edge if the sum of their weights does not exceed 1. Hence $r$ need never exceed $2 n$.

Conjecture 1.4 does not hold for all graphs, a counter example being the triangle and $\alpha_{1}=$ $\alpha_{2}=\frac{1}{2}$. Nevertheless, our proof of Theorem 1.5 holds with no change for general graphs. The previous proof of Correa and Goemans 1.4, that is cited after the statement of Conjecture 1.4, makes use of the bipartiteness of the underlying graph, but it is possible to modify their proof technique, using the results of Kano and Saito [11], such that that it works also for general graphs (see also [6]). Unlike the nonbipartite version of Theorem 1.3 (discussed in the previous paragraph), multiplicity of edges has no effect on Theorem 1.5.

\section{Edge-Coloring Weighted Bipartite Graphs}

In this section we give a proof of Theorem 1.3. The algorithm is a combination of König's Theorem [12] with the greedy algorithm. We state the König's Theorem since we use it as a subroutine in our algorithm. 
Theorem 2.1 [12] Given a bipartite (multi) graph $G=(V, E)$ there exists a coloring of edges with $\Delta=\max _{v \in V} \operatorname{deg}_{E}(v)$ colors such that all edges incident at a common vertex receive a distinct color. Moreover, such a coloring can be found in polynomial time.

The algorithm giving the guarantee of Theorem 1.3 is given in Figure 1. Observe that in Step 3 of the algorithm, $F$ can indeed be decomposed into $\lceil t n\rceil$ matchings, using Theorem 2.1 (because the maximum degree of $F$ is $\lceil t n\rceil$ ).

1. $F \leftarrow \emptyset, t \leftarrow \frac{9}{4}$.

2. Include edges in $F$ in non-increasing order of weight maintaining the property that $\operatorname{deg}_{F}(v) \leq\lceil t n\rceil$ for all $v \in V$.

3. Decompose $F$ into $r=\lceil t n\rceil$ matchings $M_{1}, \ldots, M_{r}$ and color them using colors $1, \ldots, r$. Let $F_{i} \leftarrow M_{i}$ for each $1 \leq i \leq r$.

4. Greedily add remaining edges to any of the $F_{i}$ 's maintaining that weighted degree of each color at each vertex is at most one, i.e, $\sum_{e \in \delta(v) \cap F_{i}} w_{e} \leq 1$ for each $v \in V$ and $1 \leq i \leq r$.

Figure 1: Algorithm for Edge Coloring Weighted Bipartite Graphs

We now show that the algorithm in Figure 1 gives a proper weighted coloring for $t=\frac{9}{4}$. Since the algorithm only uses $\lceil t n\rceil=\left\lceil\frac{9}{4} n\right\rceil$ colors, it is enough to show that each edge will be colored in either Step (2) when it is included in $F$ or Step (4) of the algorithm. We prove this by a series of claims which follow.

Claim 1 Each edge of weight at least $\frac{1}{t}$ is in $F$.

Proof: Let $e=\{u, v\}$ be an edge such that $w_{e} \geq \frac{1}{t}$. If $e$ cannot be added to $F$ then $\operatorname{deg}_{F}(v) \geq\lceil t n\rceil$ or $\operatorname{deg}_{F}(u) \geq\lceil t n\rceil$ when $e$ is considered in Step (2) of the algorithm. But edges added in $F$, before $e$ is considered in Step (2), have weight larger than the weight of $e$. Therefore, the total weight at the endpoint with degree at least $\lceil t n\rceil$ is at least $t n \cdot \frac{1}{t}+w_{e}>n$. A contradiction.

Lemma 2.2 If $t \geq \frac{9}{4}$ then each edge can be colored with one of the colors.

Proof: For sake of contradiction suppose some edge cannot be colored in Step (3) or Step (4). Let $e=\{u, v\}$ be such an edge and let $w_{e}=\alpha$. From Claim 1, we have that $\alpha<\frac{1}{t}$. Moreover, when $e$ is considered in Step (2), the degree of either $u$ or $v$ is already $\lceil t n\rceil$ else we would have included $e$ in $F$. Without loss of generality let that vertex be $u$, i.e, $\operatorname{deg}_{F}(u)=\lceil t n\rceil$.

For each color $1 \leq i \leq\lceil t n\rceil$, we must have that $\sum_{f \in \delta(v) \cap F_{i}} w_{f}>1-\alpha$ or $\sum_{f \in \delta(u) \cap F_{i}} w_{f}>$ $1-\alpha$ else we can color $e$ in Step (4).

Let $L_{v}=\left\{i \mid \sum_{f \in \delta(v) \cap F_{i}} w_{f}>1-\alpha\right\}$ and $k=\left|L_{v}\right|$. Then we have

$$
n>\sum_{i \in L_{v}} \sum_{f \in \delta(v) \cap F_{i}} w_{f}>k(1-\alpha)
$$

Now for each color $i \notin L_{v}$, we have $\sum_{f \in \delta(u) \cap F_{i}} w_{f}>1-\alpha$. Moreover, $\operatorname{deg}_{F}(u)=\lceil t n\rceil$ and each of these edges weighs at least $w_{e}=\alpha$. Hence, for each color $1 \leq i \leq\lceil t n\rceil$, there is an edge 
incident at $u$ colored with color $i$ with weight at least $\alpha$. Therefore

$$
\begin{aligned}
n & >\sum_{f \in \delta(u)} w_{f} \geq \sum_{1 \leq i \leq\lceil t n\rceil} \sum_{f \in \delta(u) \cap F_{i}} w_{f} \\
& =\sum_{i \in L_{v}} \sum_{f \in \delta(u) \cap F_{i}} w_{f}+\sum_{i \notin L_{v}} \sum_{f \in \delta(u) \cap F_{i}} w_{f} \\
& \geq \sum_{i \in L_{v}} \alpha+\sum_{i \notin L_{v}}(1-\alpha)=k \alpha+(\lceil t n\rceil-k)(1-\alpha) \\
& \geq k \alpha+(t n-k)(1-\alpha) .
\end{aligned}
$$

Let $\beta=\frac{k}{n}$. By scaling Inequation (1), Inequation (5) and from Claim 1, we have

$$
\begin{aligned}
\beta(1-\alpha) & <1 \\
\beta(2 \alpha-1)+t(1-\alpha) & <1 \\
\alpha & <\frac{1}{t} .
\end{aligned}
$$

We now show that for $t=\frac{9}{4}$, we have a contradiction to the above inequalities.

The expression $\beta(2 \alpha-1)+t(1-\alpha)$ is a decreasing function of $\beta$ as $2 \alpha-1<0$ since $\alpha<\frac{1}{t}<\frac{1}{2}$. Thus the expression $\beta(2 \alpha-1)+t(1-\alpha)$ has a minimum value at largest possible $\beta$ which is at most $\frac{1}{1-\alpha}$ and at $\beta=\frac{1}{1-\alpha}$, we have

$$
\beta(2 \alpha-1)+t(1-\alpha)=\frac{1}{1-\alpha}(2 \alpha-1)+t(1-\alpha)=\frac{1}{1-\alpha}-2+t(1-\alpha) .
$$

Let $g(\alpha)=\frac{1}{1-\alpha}-2+t(1-\alpha)$. We claim that $g(\alpha) \geq 1$ for each $\alpha \in\left[0, \frac{1}{t}\right)$ which gives the desired contradiction. Since, $g(\alpha)$ is a differentiable function of $\alpha$ in the range $\left[0, \frac{1}{t}\right)$ the global minimum will occur at either a local minimum or at boundary of the interval. The derivative $g^{\prime}(\alpha)=\frac{1}{(1-\alpha)^{2}}-t$. Thus the local minima can occur at $\alpha=1-\frac{1}{\sqrt{t}}$. But then

$$
\begin{aligned}
g(0) & =t-1 \geq 1 \\
g\left(\frac{1}{t}\right) & =\frac{t}{t-1}-2+t-1=\frac{(t-2)^{2}}{t-1}+1 \geq 1 \\
g\left(1-\frac{1}{\sqrt{t}}\right) & =\sqrt{t}-2+\sqrt{t}=2(\sqrt{t}-1) \geq 1
\end{aligned}
$$

where the last inequality holds for $t \geq \frac{9}{4}$. Thus $g(\alpha) \geq 1$ for each $\alpha \in\left[0, \frac{1}{t}\right)$ which contradicts inequation (7).

\section{Partitioning Bipartite Graphs}

In this section we prove Theorem 1.5. First, we give an algorithm where we show a decomposition which matches the guarantee of Correa and Goemans [5] and violates the bounds by at most two. We then show how to modify the algorithm to obtain the stronger guarantee where violation is bounded by at most one when only upper or lower bounds are present. Our algorithms use linear programming methods and the techniques have close resemblance to result of Beck and Fiala [1] on discrepancy of sets. We also note that the proofs do not use the fact that the graphs are bipartite or simple and all our results in this section also hold for general graphs with parallel edges. 
Theorem 3.1 [5] Given an instance of Balanced Decomposition of Bipartite graph problem there exists a decomposition such that

$$
\left\lfloor\alpha_{i} \operatorname{deg}_{E}(v)\right\rfloor-2 \leq \operatorname{deg}_{E_{i}}(v) \leq\left\lceil\alpha_{i} \operatorname{deg}_{E}(v)\right\rceil+2
$$

for each $1 \leq i \leq k$ and each $v \in v$.

Proof: We formulate a feasibility linear program for the following generalization of the decomposition problem. For each edge $e$, we are given a set of allowable colors $C_{e} \subseteq\{1, \ldots, k\}$ and for each vertex $v$, we have a degree bound for every color from a set of colors $K_{v} \subseteq\{1, \ldots, k\}$. We let the binary variable $x_{e}^{i}$ denote whether an edge $e$ belongs to $E_{i}$ for each edge $e \in E$ and $i \in C_{e}$. We initialize $C_{e}=\{1, \ldots, k\}, K_{v}=\{1, \ldots, k\}$ for each $v \in V$ and degree bound $B_{v}^{i}=\alpha_{i} \operatorname{deg}_{E}(v)$ for each $1 \leq i \leq k, v \in V$ which corresponds to the required decomposition in Conjecture 1.4.

$$
\begin{aligned}
\text { (LP) } & & & \\
\text { subject to } \sum_{e \in \delta(v): i \in C_{e}} x_{e}^{i} & =B_{v}^{i} & & \forall v \in V, \forall i \in K_{v} \\
\sum_{i \in C_{e}} x_{e}^{i} & =1 & & \forall e \in E \\
x_{e} & \geq 0 & & \forall e \in E
\end{aligned}
$$

Observe that the fractional solution $x_{e}^{i}=\alpha_{i}$ for each $1 \leq i \leq k$ and $e \in E$ is a fractional feasible solution to the linear program.

We give an iterative algorithm which rounds the above linear program into an integral decomposition. The integral decomposition will violate the degree bounds by an additive error of 2 giving us Theorem 3.1. The algorithm iteratively constructs the partition $E^{1}, \ldots, E^{k}$ of $E$ and is given in Figure 2.

1. Let $E^{i} \leftarrow \emptyset$ for each $1 \leq i \leq k$. While $E \neq \emptyset$ do

(a) Find an extreme point optimal solution $x$ to (LP).

(b) If there is a variable $x_{e}^{i}=0$ then remove variable $x_{e}^{i}$ and let $C_{e} \leftarrow C_{e} \backslash\{i\}$.

(c) If $x_{e}^{i}=1$ then

- $E^{i} \leftarrow E^{i} \cup\{e\}$

- $E \leftarrow E \backslash\{e\}$

- $B_{v}^{i} \leftarrow B_{v}^{i}-1$ for each $v \in e$.

(d) If there exists a vertex $v \in V$ and $1 \leq i \leq k$ such that $i \in K_{v}$ and there are at most 3 edges incident at $v$ with non-zero $x_{e}^{i}$ then remove the constraint at vertex $v$ for $i$, i.e, $K_{v} \leftarrow K_{v} \backslash\{i\}$.

2. Return $E^{i}$ for $1 \leq i \leq k$.

Figure 2: Decomposition Algorithm I

First we show that if the algorithm reaches Step (2), then the solution returned by the algorithm satisfies the guarantees claimed in Theorem 3.1. In Step (1c) we reduce $B_{v}^{i}$ whenever 
we select an edge $e$ in $E^{i}$ incident at $v$. Therefore, the bound for $E^{i}$ at any vertex $v$ can only be violated if the constraint for $v$ and $i$ is removed in Step (1d). We maintain the property that the constraint for vertex $v$ and $i$ is removed only if there are at most three edges incident at vertex $v$ which can possibly be included in $E^{i}$. Therefore, it follows that the number of edges selected in $E^{i}$ incident at $v$ is strictly less than $B_{v}^{i}+3$ and hence at most $\left\lceil B_{v}^{i}\right\rceil+2$. Moreover, we have already selected strictly more than $B_{v}^{i}-3$ edges incident at $v$ in $E^{i}$ when we remove the constraint for vertex $v$ and color $i$. Hence, the number of edges in $E^{i}$ incident at $v$ is at least $\left\lfloor B_{v}^{i}\right\rfloor-2$.

To complete the proof we show that the algorithm indeed reaches Step (2). Observe that Steps (1b), (1c) and (1d) all make progress in the sense that they reduce either the number of variables or the number of constraints. Lemma 3.2 implies that whenever Step (1a) is not applicable (because we are already at an extreme point of the current LP), at least one of these three other steps is indeed available. Since between every two applications of Step (1a) there must be an application of one of the other three steps, Step (2) must be reached eventually.

Lemma 3.2 Given an extreme point solution $x$ such that $0<x_{e}^{i}<1$ for each $e \in E$ and $i \in C_{e}$ there must exist a vertex $v$ and color $i$ satisfying the conditions of Step (1d).

Proof: Suppose for the sake of contradiction there is no vertex $v \in V$ and color $i \in K_{v}$ with at most three edges incident at $v$ with non-zero $x_{e}^{i}$. Since $x$ is an extreme point, the number of tight independent constraints equals the number of variables. We will show a contradiction to this fact by showing that the number of tight independent constraints at $x$ are strictly less than the number of variables.

We first count the number of variables. For each edge $e$, we must have $\left|C_{e}\right| \geq 2$ since $x_{e}^{i}<1$ for each edge $e$ and $i \in C_{e}$ and $\sum_{i \in C_{e}} x_{e}^{i}=1$. Hence,

$$
\text { \# of variables } \geq 2|E| \text {. }
$$

For each vertex $v \in V$ and $i \in K_{v}$, let $D_{v}^{i}$ denote the number of variables of form $x_{e}^{i}$ where $v \in e$. Since the condition of Step (1d) is not applicable we must have $D_{v}^{i} \geq 4$ for each vertex $v \in V$ and $i \in K_{v}$.

Hence,

$$
\text { \# of variables } \geq \frac{1}{2} \sum_{v \in V} \sum_{i=1}^{k} D_{v}^{i} \geq 2 \sum_{v}\left|K_{v}\right| .
$$

A simple averaging gives that the

$$
\# \text { of variables } \geq|E|+\sum_{v}\left|K_{v}\right|
$$

Observe that if equality must hold in inequations (18) and (19) then $i \in K_{v}$ whenever $i \in C_{e}$ for some $e \in \delta(v)$.

Now we bound the total number of tight independent constraints. Since $0<x_{e}^{i}<1$ for each $e$ and $i \in C_{e}$, these integrality constraints cannot be tight at $x$. The number of other constraints is exactly $|E|+\sum_{v}\left|K_{v}\right|$. Thus all of these constraints must be at equality at $x$ and linearly independent. We now show that this cannot be the case and derive a linear dependence in the tight constraints.

Summing up all the edge constraints we obtain that

$$
\sum_{e \in E} \sum_{i \in C_{e}} x_{e}^{i}=|E|
$$

where LHS is the sum of the all the variables. Summing up all the vertex constraints we obtain 


$$
\sum_{v \in V, i \in K_{v}} \sum_{e \in \delta(v): i \in C_{e}} x_{e}^{i}=\sum_{v \in V, i \in K_{v}} B_{v}^{i}
$$

where each variable occurs exactly twice in the LHS. Thus equation (21) is exactly twice of equation (20) giving us a dependence in the tight constraints which is a contradiction.

This completes the proof of the Theorem 3.1.

We now prove the second guarantee in Theorem 1.5.

Theorem 3.3 Given an instance of BALAnCEd Decomposition of Bipartite GRAPH problem, there are decompositions $F_{1}, \ldots, F_{k}$ and $G_{1}, \ldots G_{k}$ such that

$$
\begin{aligned}
& \operatorname{deg}_{F_{i}}(v) \leq\left\lceil\alpha_{i} \operatorname{deg}_{E}(v)\right\rceil+1 \\
& \operatorname{deg}_{G_{i}}(v) \geq\left\lfloor\alpha_{i} \operatorname{deg}_{E}(v)\right\rfloor-1
\end{aligned}
$$

for each $1 \leq i \leq k$ and $v \in V$.

Proof: We first show how to construct the decomposition $F_{1}, \ldots, F_{k}$ which satisfies the upper bounds within an additive error of 1 . The algorithm is very similar to the algorithm given in Figure 2 with the following difference. The relaxation step (1d) is modified and the constraint for $i \in K_{v}$ is removed whenever $D_{v}^{i} \leq\left\lceil B_{v}^{i}\right\rceil+1$ where $D_{v}^{i}$ is the number of variables of the form $x_{e}^{i}$ for some edge $e$ incident at $v$.

1. Let $F^{i} \leftarrow \emptyset$ for each $1 \leq i \leq k$. While $E \neq \emptyset$ do

(a) Find an extreme point optimal solution $x$ to (LP).

(b) If there is a variable $x_{e}^{i}=0$ then remove variable $x_{e}^{i}$ and let $C_{e} \leftarrow C_{e} \backslash\{i\}$.

(c) If $x_{e}^{i}=1$ then

- $F^{i} \leftarrow F^{i} \cup\{e\}$

- $E \leftarrow E \backslash\{e\}$

- $B_{v}^{i} \leftarrow B_{v}^{i}-1$ for each $v \in e$.

(d) If there exists a vertex $v \in V$ and $i$ such that $i \in K_{v}$ and $D_{v}^{i} \leq\left\lceil B_{v}^{i}\right\rceil+1$ then remove the constraint at vertex $v$ for color $i$, i.e, $K_{v} \leftarrow K_{v} \backslash\{i\}$. Here $D_{v}^{i}=\mid\left\{e \in \delta(v): x_{e}^{i}>\right.$ $0\} \mid$.

2. Return $F^{i}$ for $1 \leq i \leq k$.

Figure 3: Decomposition Algorithm II

If the modified algorithm reaches Step (2) then it gives the claimed guarantee since the bound for color $i$ at vertex $v$ is violated only when the corresponding constraint is removed in Step (1d). In such a case we have $D_{v}^{i} \leq\left\lceil B_{v}^{i}\right\rceil+1$ and hence the total number of edges in $F^{i}$ incident at $v$ are bounded by $\left\lceil B_{v}^{i}\right\rceil+1$ as desired.

To complete the proof of the Theorem 3.3 we show that the algorithm reaches Step (2). As in the discussion preceding Lemma 3.2, this will follow from the following lemma. 
Lemma 3.4 Given an extreme point solution $x$ such that $0<x_{e}^{i}<1$ for each $v \in V$ and $i \in K_{v}$ there must exist a vertex $v$ and color $i$ such that $D_{v}^{i} \leq\left\lceil B_{v}^{i}\right\rceil+1$ where $D_{v}^{i}$ is the number of variables of the form $x_{e}^{i}$ for some edge e incident at $v$.

Proof: Suppose for sake of contradiction we have $D_{v}^{i} \geq\left\lceil B_{v}^{i}\right\rceil+2$ for each $i \in K_{v}$. We give a contradiction to the fact that the number of tight independent constraints is equal to the number of variables in $x$.

The contradiction is shown by a counting argument. We give one token to each variable which redistributes its token to the constraints. We then collect one token for each tight independent constraint and still have extra tokens, giving us the contradiction. The redistribution is given by the following two rules.

- Rule 1: Each variable $x_{e}^{i}$ gives $x_{e}^{i}$ tokens to the constraint for edge $e$.

- Rule 2: Each variable $x_{e}^{i}$ gives $\frac{1-x_{e}^{i}}{2}$ tokens to the constraint for each endpoint $v$ of $e$ and $i$.

Observe that each edge gives a total of one token.

Now, we count the number of tokens received by each constraint. Edge constraint for an edge $e$ receives $\sum_{i \in C_{e}} x_{e}^{i}$ tokens from Rule 1 which is exactly one from the edge constraint of $e$ in (LP). Hence, each edge constraint receives one token in the redistribution.

Consider a constraint for vertex $v \in V$ and $i \in K_{v}$. It receives $\frac{1-x_{e}^{i}}{2}$ tokens for each $e \in \delta(v)$ such that $x_{e}^{i}>0$ by Rule 2 or equivalently each edge counting towards $D_{v}^{i}$. Hence, the total number of tokens received by the constraint is at least

$$
\begin{aligned}
\sum_{e \in \delta(v), x_{e}^{i}>0} \frac{1-x_{e}^{i}}{2} & =\frac{1}{2}\left(D_{v}^{i}-\sum_{e \in \delta(v), x_{e}^{i}>0} x_{e}^{i}\right) \\
& \geq \frac{1}{2}\left(D_{v}^{i}-B_{v}^{i}\right) \geq 1
\end{aligned}
$$

where the last inequality follows since $D_{v}^{i} \geq B_{v}^{i}+2$. Thus each degree constraint also receives at least one token. Moreover, if any of the constraints receives more than token or there is a vertex $v \in V$ and color $i \notin K_{v}$ such that $x_{e}^{i}>0$ for some edge $e \in \delta(v)$ then $\frac{1-x_{e}^{i}}{2}$ token given by Rule 2 is extra and gives us the contradiction. Otherwise, for any color $i$ and vertex $v$, we must have that $i \in K_{v}$ whenever $i \in C_{e}$. But then the sum of all the edge constraints exactly equals the sum of the all the degree constraints, contradicting the requirement that the constraints are linearly independent.

This completes the proof that there exists a decomposition $F_{1}, \ldots, F_{k}$ satisfying the upper bounds within additive error of one.

We now show how to construct the decomposition $G_{1}, \ldots, G_{k}$ which satisfies the lower bounds within an additive error of one. The algorithm is exactly similar to one in Figure 3 except that we modify Step (1d) in the following manner. We delete the constraint for vertex $v \in V$ and $i \in K_{v}$ only when $\left\lfloor B_{v}^{i}\right\rfloor \leq 1$. Observe that with this modification it is easy to verify that the solution returned satisfies the lowerbound within an additive error of 1 . This follows from the fact that at least $\left\lfloor B_{v}^{i}\right\rfloor-1$ edges incident at $v$ are in $G_{i}$ before we remove the degree constraint for vertex $v$ and color $i$.

We now show that algorithm will make progress with the modified Step (1d).

Lemma 3.5 Given an extreme point solution $x$ such that $0<x_{e}^{i}<1$ for each $v \in V$ and $i \in K_{v}$ there must exist a vertex $v$ and color $i$ such that $\left\lfloor B_{v}^{i}\right\rfloor \leq 1$. 
Proof: Suppose for sake of contradiction we have $\left\lfloor B_{v}^{i}\right\rfloor \geq 2$ for each $i \in K_{v}$. We give a contradiction to the fact that number of tight independent constraints equal the number of variables in $x$.

The contradiction is shown by a counting argument. We give one token to each variable which redistributes its token to the constraints such that we can collect one token for each tight independent constraints and still have extra tokens to spare. The redistribution is given the following two rules.

1. Rule 1: Each variable $x_{e}^{i}$ gives $1-x_{e}^{i}$ tokens to the constraint for edge $e$.

2. Rule 2: Each variable $x_{e}^{i}$ gives $\frac{x_{e}^{i}}{2}$ tokens to the constraint for $i$ and $v$ where $e \in \delta(v)$.

Observe that each edge gives a total of one token. Now, we count the tokens received by each constraint. The constraint for an edge $e$ receives $\sum_{i \in C_{e}}\left(1-x_{e}^{i}\right)=\left(\sum_{i \in C_{e}} 1\right)-1=$ $\left|C_{e}\right|-1 \geq 1$ tokens since $\left|C_{e}\right| \geq 2$. Hence, each edge constraint receives at least one token in the redistribution. Now, consider a constraint for $v$ and $i \in K_{v}$. It receives $\frac{x_{e}^{i}}{2}$ token for each $e \in \delta(v)$ such that $x_{e}^{i}>0$. Hence, the total tokens received by the constraint is at least

$$
\sum_{e \in \delta(v): i \in C_{e}} \frac{x_{e}^{i}}{2}=\frac{B_{v}^{i}}{2} \geq 1
$$

where the last inequality follows from the fact that $B_{v}^{i} \geq 2$. Thus each constraint receives at least one token. Moreover, if any of the constraints receives more than one token or there is a vertex $v \in V$ and $i \notin K_{v}$ such that $x_{e}^{i}>0$ for some edge $e \in \delta(v)$ then $\frac{x_{e}^{i}}{2}$ token from Rule 2 is extra giving us the contradiction. Hence for any color $i$ and vertex $v$, we must have that $i \in K_{v}$ whenever $i \in C_{e}$. Moreover, all the constraints must be linearly independent. But then the sum of all the edge constraints exactly equals the sum of the all the degree constraints giving us a contradiction to independence of all constraints.

This completes the proof of Theorem 3.3.

\section{Acknowledgements}

Part of this work was performed at Microsoft Research, Redmond, Washington.

\section{References}

[1] J. Beck and T. Fiala,"Integer-Making" Theorems, Discrete Applied Mathematics, 3 (1981), pp. 1-8.

[2] John Beetem, Monty Denneau and Don Weingarten, The GF11 Supercomputer, Proceedings of the 12th annual international symposium on Computer architecture, p.108-115, June 17-19, 1985, Boston, Massachusetts, United States.

[3] Shun-Ping Chung and Keith W. Ross, On Nonblocking Multirate Interconnection Networks, SIAM Journal of Computing, volume 20:4, pages 726-736, 1991.

[4] C. Clos, A Study of Nonblocking Switching Networks, Bell System Technical Journal, vol. 32, no. 2, pp. 406-424, 1953. 
[5] J. Correa and M.X. Goemans, Improved Bounds on Nonblocking 3-Stage Clos Networks, SIAM Journal of Computing, 37, pp. 870-894, 2007.

[6] J.R. Correa and M. Matamala, Some Remarks About Factors of Graphs, Journal of Graph Theory, 57:265-274, 2008.

[7] D. de Werra, On Some Combinatorial Problems arising in Scheduling, Operations Research Society Journal 8, 165-175, 1970.

[8] D. Z. Du , B. Gao, F. K. Hwang and J. H. Kim, On Multirate Rearrangeable Clos Networks, SIAM Journal on Computing, v.28 n.2, p.463-470, April 1999.

[9] A. J. Hoffman, Generalization of a theorem of Knig, Journal of the Washington Academy of Science 46, 211-212, 1956.

[10] A. Itoh, W. Takahashi, H. Nagano, M. Kurisaka and S. Iwasaki, Practical Implementation and Packaging Technologies for a Large-Scale ATM Switching System, Journal of Selected Areas in Communications 9, 1280-1288, 1991.

[11] M. Kano and A. Saito, [a,b]-factors of graphs, Discrete Mathematics, 47, pages 113-116, 1983.

[12] D. König, Graphok s Alkalmazsuk a Determinnsok s a Halmazok Elmletre, Mathematikai s Termszettudomnyi rtesit 34, 104-119, 1916.

[13] Guo-Hui Lin , Ding-Zhu Du , Xiao-Dong Hu and Guoliang Xue, On Rearrangeability of Multirate Clos Networks, SIAM Journal on Computing, v.28 n.4, p.1225-1231, Aug. 1999.

[14] G. Lin, D. Du, W. Wu and K. Yoo, On 3-Rate Rearrangeability of Clos Networks, DIMACS Series in Discrete Mathematics and Theoretical Computer Science Vol 42, 315-333, 1998.

[15] Riccardo Melen and Jonathan S. Turner, Nonblocking Multirate Networks, SIAM Journal on Computing, v.18 n.2, p.301-313, April 1989.

[16] Hung Q. Ngo and Van H. Vu, Multirate Rearrangeable Clos Networks and a Generalized Edge Coloring Problem on Bipartite Graphs, Proceedings of the fourteenth annual ACMSIAM symposium on Discrete algorithms, January 12-14, 2003, Baltimore, Maryland.

[17] D. Slepian, Two Theorems on a Particular Crossbar Switching, unpublished manuscript, 1958.

[18] V. G. Vizing. On an Estimate of the Chromatic Class of a p-Graph (in Russian). Diskret. Analiz, 3:23-30, 1964. 